\title{
Metrics for Characterization of Atom Probe Instrument Performance
}

\author{
Ian M. Anderson, ${ }^{*}$ Eric B. Steel, ${ }^{*}$ and Karen Torres Henry* \\ * Surface and Microanalysis Science Division, National Institute of Standards and Technology, \\ 100 Bureau Dr., Gaithersburg MD 20899-8371 ian.anderson@nist.gov
}

Atom probe field ion microscopy long served as a powerful technique for the characterization of metals and alloys at sub-nanometer spatial resolution [1], a role that became more powerful with the development of three-dimensional data acquisition in its present incarnation as atom probe tomography (APT) [2]. Subsequent developments in atom probe instrumentation over the past decade, such as the miniaturization of the specimen-electrode assembly and the introduction of laser pulsing as an alternative to voltage pulsing [3], have broadened the applications of APT beyond metallurgy [4], but with concomitant complexity in data reconstruction, analysis, and interpretation [5]. The relative simplicity for APT data analysis of metallurgical specimens arises from the special properties of metals, for example their high electrical and thermal conductivities and the directional isotropy of metal bonding. In contrast, carbon-based materials, including organic matter, tend to exhibit highly directional covalent bonds, and nanoelectronic devices are comprised of materials whose electrical conductivities vary by as much as twenty orders of magnitude. Substantial improvements in post acquisition analysis, or new developments in data acquisition that intrinsically mitigate these complications, will be required before APT can make contributions comparable to those in metallurgy to such fields as electronics or biology.

A first step toward more sophisticated APT data analysis is the robust characterization of instrument performance. Accordingly, we have begun to develop quantifiable performance metrics and standard measurement protocols for atom probe instrumentation. Such metrics are useful both for monitoring the performance of a given instrument over time and for the comparison and conversion of operating parameters among different instruments, which may feature substantially different architectures. We have begun by devising metrics using the pure aluminum specimen traditionally used for measuring mass spectral resolution. This specimen has several advantages: it is easily and routinely fabricated, and runs well in the instrument; it has a simple mass spectrum, being comprised of a single element that has only one stable isotope; it has a relatively low evaporation field, so that a large field of view can be achieved with a correspondingly low applied standing voltage; and it exhibits a strong texture when fabricated from drawn wire, so that the specimen exhibits a crystallographic contrast centered on a low-index zone axis, such as [001].

A metric for the available angular field of view (FoV) takes advantage of the [001] texture of the drawn aluminum wire. Four nearby $<013>$ poles, and the $\{331\}$ zone lines that connect them, form an easily identifiable near-square pattern centered on the [001] pole, as shown in the Fig. 1a. The available angular FoV can be determined by the area of the stereographic micrograph exhibiting crystallographic intensity relative to that of a circle that passes through the $<013>$ poles, which subtends a linear axial angle of $2 \theta=36.9^{\circ}$, with $\theta$ expressed in the $(r, \theta, \varphi)$ polar coordinate system, and a solid angle, $\Omega=2 \pi(1-\cos \theta)$ of $\Omega=0.32 \mathrm{sr}$. Current generation atom probe instruments exhibit an angular FoV of $\Omega=0.5 \mathrm{sr}$ or $2 \theta=46^{\circ}$, with evident intensity from a circle of radius $1.25 \times$ larger than that intersecting the $<013>$ poles, as illustrated in Fig. 1. 
Instruments with different specimen / counter electrode geometries may exhibit as much as a factor of two difference in the applied voltage necessary to achieve a given evaporation field at the specimen. This voltage-field relationship can be quantified as a characteristic reference voltage, $\mathrm{V}_{\text {ref, }}$ which can be defined as the voltage at which the aluminum specimen exhibits an easily characterized FoV. The choice of standing voltage, $\mathrm{V}_{\mathrm{s}}$, for an experiment with a different instrument would then be adjusted by the corresponding ratio of $\mathrm{V}_{\text {ref }}$ between instruments.

The measurement of mass resolution is traditionally performed at the singly charged $\mathrm{Al}^{+}$ion at a nominal mass $(\mathrm{m})$ to charge $(q)$ ratio of $m / q=27 \mathrm{amu}$. Because the mass resolution decreases with increasing field of view due to trajectory aberrations, these measurements must be specified as performed at some standard fraction (or as a function of) the reference voltage, $\mathrm{V}_{\text {ref. }}$. The intensity in the tails of the mass spectral peaks play an important role in establishing detection limits of dilute solutes; accordingly, the full width at half (FWHM), tenth (FWTM) and hundredth (FW1\%M) maximum are typically used to characterize mass resolution, as shown in Fig. 1b. However, these metrics do not describe the evident asymmetric shape of individual mass peaks, which are typically skewed to the high mass side, and which may substantially differ between voltage- and laser-pulsing modes. A probability distribution function that captures the variation in the shape of the spectral peaks would be more suitable. The variation in peak shape should ideally be characterized across the full mass spectrum; a suitable multi-element standard with well separated spectral peaks should be identified for this purpose, and could also serve as a calibration standard for the mass spectrum.

[1] M.K. Miller et al., Atom Probe Field Ion Microscopy, Clarendon Press, Oxford, UK (1996).

[2] M.K. Miller, Atom Probe Tomography, Kluwer Academic / Plenum, New York, NY (2000).

[3] T.F. Kelly and M.K. Miller, Rev. Sci. Instrum. 78 (2007) 031101.

[4] D.N. Seidman and K. Stiller (ed.), A Renaissance in APT, MRS Bulletin 34 (2009) No. 10.

[5] G. Schmitz (ed.), Proc. IFES 2010, Ultramicroscopy (2011) in press.
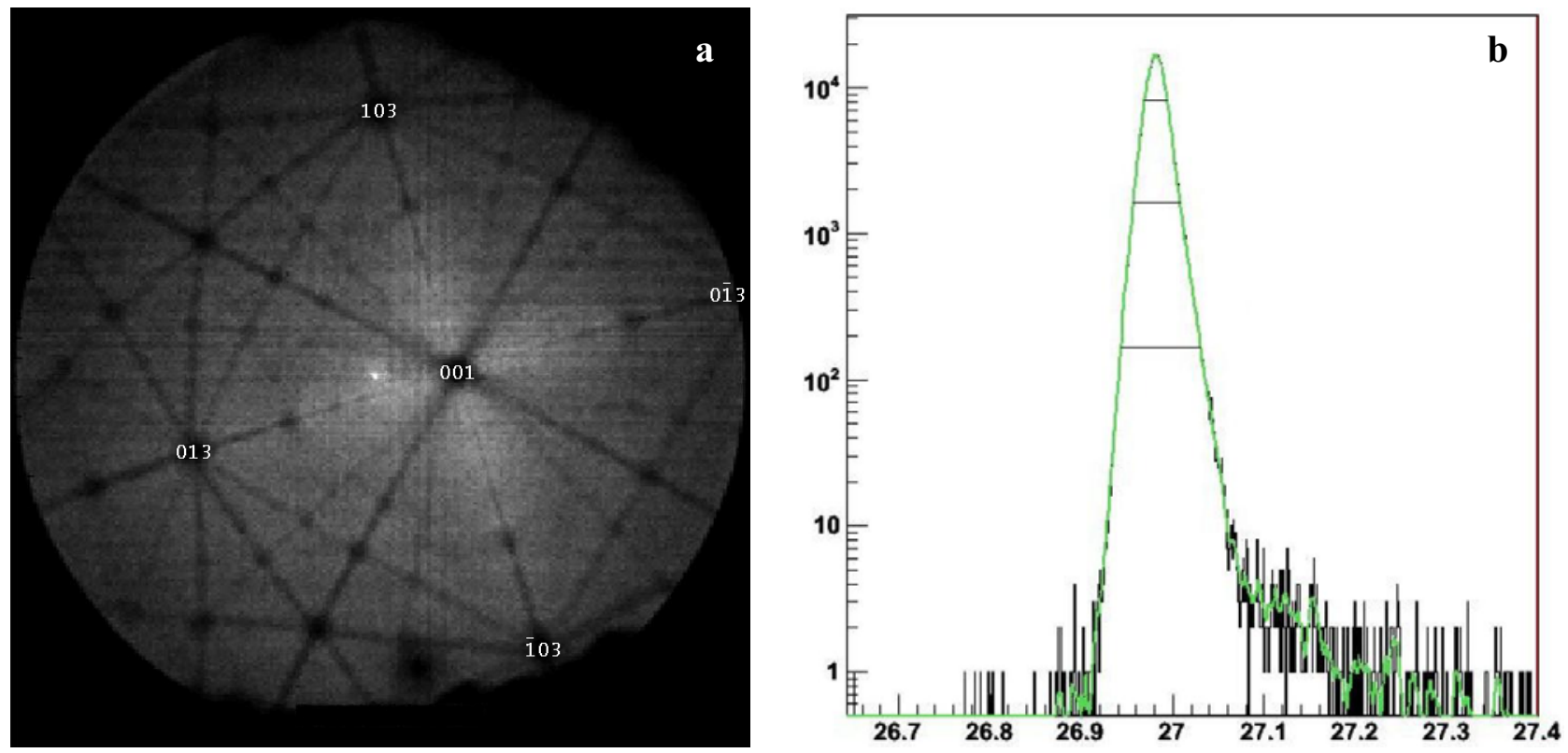

FIG. 1. Representative APT data acquired from an [001]-oriented pure aluminum specimen: (a) field evaporation histogram exhibiting three of four $<013>$-type poles within the detectable field of view; (b) mass spectrum near $m / q=27$ with FWHM, FWTM, and FW1\%M indicated. 